\title{
THE MUTUAL INDUCTANCE OF TWO PARALLEL CIRCLES
}

\author{
By Chester Snow
}

\section{ABSTRACT}

It is shown that the mutual inductance of two parallel circles is identical with the electrostatic potential due to a fairly simple surface charge upon an annular surface. This potential is formulated as a complex line integral, and by deformation of the path its harmonic expansion is obtained for all possible cases.

\section{CONTENTS}

I. Introduction

II. The equivalent electrostatic problem

III. A complex-integral representation of the mutual inductance._._._- 259

IV. Formula when the distance between centers is greater than the sum of

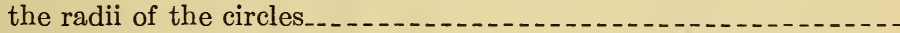

V. Formula when the distance between centers is less than the difference of the radii .

VI. Formula when the distance between centers is greater than the difference and less than the sum of the radii

VII. Summary ..............

\section{INTRODUCTION}

If a fixed circle of radius $a_{1}$ lies in the $y, z$ plane with center at the origin, the position of a second circle parallel to the first whose radius is $a_{2}$ may be specified either by the cylindrical coordinates $x, \rho, \phi$, or the spherical coordinates $r, \theta, \phi$, of its center $P$. It is an interesting fact that the mutual inductance $M(x, \rho)$ (independent of $\phi$ ) of the two circles satisfies Laplace's equation in the coordinates of $P$, and $M$ may, therefore, be identified with the electrostatic potential due to a certain distribution of electricity. This was pointed out by Butterworth ${ }^{1}$ who did not determine the equivalent distribation of electrostatic charge, but who made use of the fact that $\nabla^{2} M=0$, to find the expansion for $M$, in zonal harmonics for the case where $r>a_{1}+a_{2}$. For such a type of expansion there are, in fact, three cases to be considered according as the second circle, when brought into the plane of the first by keeping the distance between centers fixed, intersects the first circle or falls wholly within or without it. Butterworth's formula for the case where $r>a_{1}+a_{2}$ is complete, and his treatment of the case of equal circles when $r<2 a$ would be so except for the fact that he obtained only the first four terms of the expansion. The same objection applies to his treatment of the case of "unequal circles close together." Moreover, the expression for the

1 Butterworth, Phil. Mag., 31, p. 443; 1916. 
mutual inductance of coaxial circles upon which Butterworth founds his solution in this case is an expansion of the type

$$
M(x, o)=\sum_{k=0}^{\infty}\left[1+\frac{x^{2}}{\left(a_{1}-a_{2}\right)^{2}}\right]^{k}\left[A_{k} \log \left(1+\frac{x^{2}}{\left(a_{1}-a_{2}\right)^{2}}\right)+B_{k}\right]
$$

and it is easy to show that such an expression can not be valid for the entire range $a_{1}-a_{2}<x<a_{1}+a_{2}$, and indeed, if $\frac{a_{2}}{a_{1}}<3-2 \sqrt{2}=0.172$, it can never be valid, but there must be negative powers of $1+\frac{x^{2}}{\left(a_{1}-a_{2}\right)^{2}}$ in the expansion.

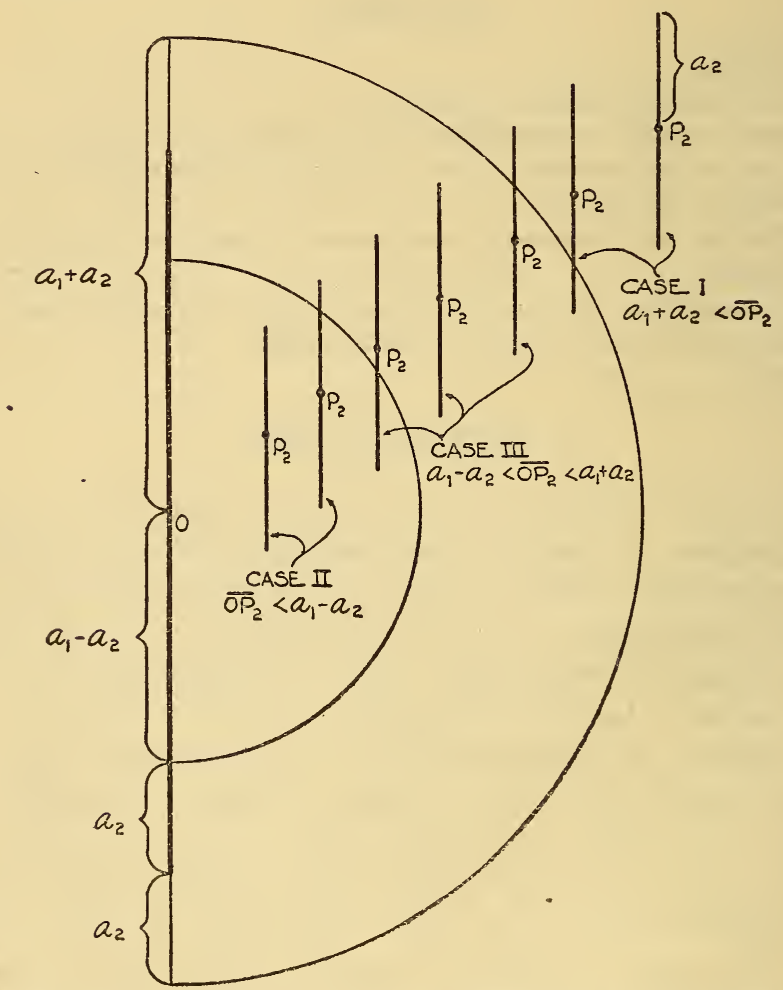

FIgURE 1.-Showing various relative positions of the small circle (whose center is $P_{2}$ ) with respect to larger one whose center is at 0

Butterworth tested his solution for this case by showing that it would (if completely carried out) give the correct solution for equal circles and also in some cases not clearly defined when the circles were unequal, but coplanar. That these tests are not crucial may be seen from the presence of a series in the solution about to be given of the type $\sum_{k=1}^{\infty} A_{k}\left(\frac{a_{1}-a_{2}}{r}\right)^{2 k} P_{2 k-1}(\cos \theta)$ which vanishes when the 
circles are equal $\left(a_{1}=a_{2}\right)$, and also when they are unequal but coplanar, thus satisfying both the above tests without vanishing in general.

In the following it is shown that the mutual inductance $M(r, \mu)$ of the two circles, the center of the smaller being at the point $r, \mu(=\cos \theta)$, is identical with the electrostatic potential due to a symmetrical surface charge of electricity, of total amount zero, upon the annulus in the plane $x=0$ whose boundaries are the two concentric circles $\rho=a_{1}+a_{2}$ and $\rho=a_{1}-a_{2}$ where $\rho^{2}=y^{2}+z^{2}$. The surface density $\sigma$ of this distribution is found to be a simple function of $\rho$, equation (10), and its Newtonian potential is expressed as a complex line integral. By deforming the path of this integral the residues of the poles of the integrand give the zonal harmonic development of this potential for the various cases. The three possible cases are shown in Figure 1.

\section{THE EQUTVALENT ELECTROSTATIC PROBLEM}

It has been shown by the writer ${ }^{2}$ that the mutual inductance of any two parallel circles is given by the equation.

$$
M=4 \pi^{2} a_{1} a_{2} \int_{0}^{\infty} e-s x J_{0}(\rho s) J_{1}\left(a_{1} s\right) J_{1}\left(a_{2} s\right) d s
$$

If we make use of Gegenbauer's formula ${ }^{3}$

$$
J_{1}\left(a_{1} s\right) J_{1}\left(a_{2} s\right)=\frac{a_{1} a_{2} s}{\pi} \int_{0}^{\pi} \frac{J_{1}\left(s \rho_{1}\right)}{\rho_{1}} \sin ^{2} \psi d \psi
$$

where $\rho_{1}$ is defined by

$$
\rho_{1}^{2}=a_{1}^{2}-2 a_{1} a_{2} \cos \psi+a_{2}^{2}
$$

the expression (1) becomes

$$
\begin{aligned}
M & =4 \pi a_{3}^{2} a_{2}^{2} \int_{0}^{\infty} s e^{-s x} J_{0}(\rho s) d s \int_{0}^{\pi} \frac{J_{1}(s \rho)}{\rho_{1}} \sin ^{2} \psi d \psi \\
& =4 \pi a_{1}^{2} a_{2}^{2} \int_{0}^{\pi} \frac{\sin ^{2} \psi d \psi}{\rho_{1}} \int_{0}^{\infty} s e^{-s x} J_{0}(\rho s) J_{1}\left(\rho_{1} s\right) d s \\
& =-4 \pi a_{1}^{2} a_{2}^{2} \int_{0}^{\pi} \frac{\sin ^{2} \psi d \psi}{\rho_{1}} \frac{\partial}{\partial \rho_{1}} \int_{0}^{\infty} e^{-s x} J_{0}(\rho s) J_{0}\left(\rho_{1} s\right) d s
\end{aligned}
$$

If we use $\rho_{1}$ instead of $\psi$ for the variable of integration, then by equation (3)

and

$$
-4 \pi a_{1}^{2} a_{2}^{2} \frac{\sin ^{2} \psi d \psi}{\rho_{1}}=-2 \pi \sqrt{\left[\left(a_{1}+a_{2}\right)^{2}-\rho_{1}^{2}\right]\left[\rho_{1}^{2}-\left(a_{1}-a_{2}\right)^{2}\right]} d \rho_{1}
$$

$M=-2 \pi \int_{a_{1}-a_{2}}^{a_{1}+a_{2}} \sqrt{\left[\left(a_{1}+a_{2}\right)^{2}-\rho_{1}^{2}\right]\left[\rho_{1}^{2}-\left(a_{1}-a_{2}\right)^{2}\right]} d \rho_{1} \frac{\partial}{\partial \rho_{1}} \int_{0}^{\infty} e^{-s x} J_{0}\left(\rho_{1} s\right) J_{0}\left(\rho_{1} s\right) d s$

2 B. S. Jour. Research, 1, p. 537; 1928.

3 N. Nielsen, Handbuch der Theorie der Cylinderfunktionen, p. 183. 
Integrating by parts (with respect to $\rho_{1}$ ) gives

$$
\begin{gathered}
M=2 \pi \int_{a_{1}-a_{2}}^{a_{1}+a_{2}} d \rho_{1}\left(\frac{d}{d \rho_{1}} \sqrt{\left[\left(a_{1}+a_{2}\right)^{2}-\rho_{1}^{2}\right]\left[\rho_{1}^{2}-\left(a_{1}-a_{2}\right)^{2}\right]}\right) . \\
\cdot \int_{0}^{\infty} e^{-s x} J_{0}(\rho s) J_{0}\left(\rho_{1} s\right) d s
\end{gathered}
$$

Now from the well-known integral formula

If we now let

$$
\int_{0}^{\infty} e^{-s x} J_{0}(s R) d s=\frac{1}{\sqrt{x^{2}+R^{2}}}
$$

$$
R^{2}=\rho^{2}-2 \rho \rho_{1} \cos \left(\phi-\phi_{1}\right)+\rho_{1}^{2}
$$

then by the use of Neumann's addition formula

$$
J_{0}(s R)=J_{0}(\rho s) J_{0}\left(\rho_{1} s\right)+2 \sum_{n=1}^{\infty} J_{n}(\rho s) J_{n}\left(\rho_{1} s\right) \cos n\left(\phi-\phi_{1}\right)
$$

it follows that

$$
\begin{gathered}
\frac{1}{\sqrt{x^{2}+\rho^{2}-2 \rho \rho_{1} \cos \left(\phi-\phi_{1}\right)+\rho_{1}^{2}}}= \\
=\int_{0}^{\infty} e^{-x s} J_{0}(\rho s) J_{0}\left(\rho_{1} s\right) d s+2 \sum_{n=1}^{\infty} \cos n\left(\phi-\phi_{1}\right) \int_{0}^{\infty} e^{-s x} J_{n}(\rho s) J_{n}\left(\rho_{1} s\right) d s
\end{gathered}
$$

If this equation be multiplied by $d \phi_{1}$ and integrated from 0 to $2 \pi$, it gives

$$
2 \pi \int_{0}^{\infty} e^{-x s} J_{0}(\rho s) J_{0}\left(\rho_{1} s\right) d s=\int_{0}^{2 \pi} \frac{d \phi_{1}}{\sqrt{x^{2}+\rho^{2}-2 \rho \rho_{1} \cos \left(\phi-\phi_{1}\right)+\rho_{1}^{2}}}
$$

By the aid of this, the expression (4) for $M$ may be written

$$
M=\int_{a_{1}-a_{2}}^{a_{1}+a_{2}} d \rho_{1} \int_{0}^{2 \pi} \frac{\rho_{1} \sigma\left(\rho_{1}\right) d \phi_{1}}{\sqrt{x^{2}+\rho^{2}-2 \rho \rho_{1} \cos \left(\phi-\phi_{1}\right)+\rho_{1}^{2}}}
$$

where if

$$
\begin{gathered}
a_{1}-a_{2}<\rho_{1}<a_{1}+a_{2} \\
\sigma\left(\rho_{1}\right)=\frac{1}{\rho_{1}} \frac{d}{d \rho_{1}} \sqrt{\left[\left(a_{1}+a_{2}\right)^{2}-\rho_{1}^{2}\right]\left[\rho_{1}^{2}-\left(a_{1}-a_{2}\right)^{2}\right]}
\end{gathered}
$$

or

$$
\rho_{1} \sigma\left(\rho_{1}\right)=\frac{2\left(a_{1}^{2}+a_{2}^{2}-\rho_{1}^{2}\right) \rho_{1}}{\sqrt{\left[\left(a_{1}+a_{2}\right)^{2}-\rho_{1}^{2}\right]\left[\rho_{1}^{2}-\left(a_{1}-a_{2}\right)^{2}\right]}}
$$

The radical in the integrand of the double integral (9) represents the distance from the point of integration $P_{1}$, whose cylindrical coordinates are $x=0, \rho=\rho_{1}, \phi=\phi_{1}$, to the general point $P(x, \rho, \phi)$, and the 
integration extends over an annulus in the $y, z$ plane which is bounded by the concentric circles $\rho_{1}=a_{1}-a_{2}$ and $\rho_{1}=a_{1}+a_{2}$. Since $\sigma$ is a function of $\rho_{1}$ only (not involving $\phi_{1}$ ), the integral is independent of $\phi$, and we recognize in this integral the Newtonian potential at $P$ due to a symmetrical electrostatic charge on this annulus whose surface density $\sigma$ is the function of $\rho_{1}$ defined by equation (10). Although this density becomes infinite at the inner and outer edges of the annulus, it does so in such a manner that its Newtonian potential is everywhere finite and continuous. This latter vanishes at infinity to the order of $\frac{1}{r^{3}}$, since the total charge $2 \pi \int_{a_{1}+a_{2}}^{a_{1}+a_{2}} \rho_{1} \sigma d \rho_{1}$ is zero. This establishes the identity of the mutual inductance of the circles and the electrostatic potential. When the point $P$ comes on to the charged annulus, this corresponds to the two circles becoming coplanar and intersecting.

\section{A COMPLEX-INTEGRAL REPRESENTATION OF THE MUTUAL INDUCTANCE}

If we multiply together the binominal series for

$\left[1-\left(\frac{\rho_{1}}{a_{1}+a_{2}}\right)^{2}\right]^{-\frac{1}{2}}$ and for $\left[1-\left(\frac{a_{1}-a_{2}}{\rho_{1}}\right)^{2}\right]^{-\frac{1}{2}}$ and then rearrange the resulting double series in ascending and descending powers of $\rho_{1}$, we find an expansion for the function $\rho_{1} \sigma\left(\rho_{1}\right)$ given by equation (10). Since $\rho_{1}$ is a value of $r$ when $\theta=\frac{\pi}{2}$ that is, $\mu=\cos \theta=0$-we may replace it by $r$, and the expansion is

$$
\begin{aligned}
-\left[\frac{\partial M(r, \mu)}{\partial \mu}\right]_{\mu=+0} & =2 \pi r \sigma(r)=\left(a_{1}+a_{2}\right) \sum_{k=-\infty}^{k=+\infty}\left(k+\frac{1}{2}\right) b_{k}(\lambda)\left(\frac{r}{a_{1}+a_{2}}\right)^{2 k} \\
& \quad \text { if } a_{1}-a_{2}<r<a_{1}+a_{2} \\
= & o \text { if } r<a_{1}-a_{2} \text { or if } r>a_{1}+a_{2}
\end{aligned}
$$

where for general values of $\nu$, the function of $\nu$ and $\lambda, b_{\nu}(\lambda)$ is defined by

$$
\begin{gathered}
b_{\nu}(\lambda) \equiv \frac{\Gamma\left(-\frac{1}{2}\right) \Gamma\left(\nu-\frac{1}{2}\right)}{\Gamma(\nu+1)} F\left(-\frac{1}{2}, \nu-\frac{1}{2}, \nu+1, \lambda\right) \\
=\sum_{s=0}^{\infty} \frac{\Gamma\left(s-\frac{1}{2}\right) \Gamma\left(s+\nu-\frac{1}{2}\right)}{\Gamma(s+1) \Gamma(s+\nu+1)} \lambda^{s} \\
\lambda \equiv\left(\frac{a_{1}-a_{2}}{a_{1}+a_{2}}\right)^{2}
\end{gathered}
$$


where $F$ denotes the hypergeometric function. It follows from this definition that when (and only when) $\nu=k=$ an integer (positive, negative or zero), $b_{\nu}$ satisfies the following equation:

$$
b_{k}(\lambda)-\lambda^{-k} b_{-k i}(\lambda)=0
$$

It is evident from the definition (13) of $b_{\nu}(\lambda)$ as a function of the complex variable $\nu$ (with $\lambda$ fixed and less than one) that $b_{\nu}$ is analytic at all finite points of the $\nu$ plane except for the simple poles at $\nu=\frac{1}{2}-k$ where $k=0,1,2,3 \cdots$ Similarly, the poles of $b_{-\nu}$ are at $\nu=k-\frac{1}{2}$. Consequently all the poles of $b_{\nu}$ and $b_{-\nu}$ are the totality of zeros of the function $\cos \nu \pi$ and, therefore $b_{\nu} \cos \nu \pi$ and $b_{-\nu} \cos \nu \pi$ have no poles and $\cos \nu \pi\left(b_{\nu}-\lambda^{-\nu} b_{-\nu}\right)$ has no poles but vanishes whenever $\nu$ is a real integer; that is, for all points at which $\sin \nu \pi$ vanishes. Consequently the function $g_{\nu}(\lambda)$ defined by

$$
g_{\nu}(\lambda) \equiv-\pi \cot \nu \pi\left(b_{\nu}(\lambda)-\lambda^{-\nu} b_{-\nu}(\lambda)\right)
$$

is analytic at all finite points in the $\nu$ plane. It does not vanish at the poles of $b_{\nu}$ and $b_{-\nu}$, nor at the poles of $\cot \nu \pi$. This function so defined satisfies identically the equation

$$
g_{\nu}(\lambda)-\lambda^{-\nu} g_{-\nu}(\lambda) \equiv 0
$$

for all values of $\nu$, whereas the corresponding equation (14) for $b_{\nu}$ only holds when $\nu$ is integral.

We find a simpler expression for the function $g_{\nu}(\lambda)$ by applying Gauss' transformation to the hypergeometric function $F\left(\frac{3}{2}, \nu+\frac{3}{2}\right.$, $3,1-\lambda)$, which gives

$$
\begin{aligned}
g_{\nu}(\lambda) & =\frac{\pi^{2}}{2}(1-\lambda)^{2} F\left(\frac{3}{2}, \nu+\frac{3}{2}, 3,1-\lambda\right) \\
& =8 \pi^{2} \frac{a_{2}^{2}}{a_{1}^{2}}\left(\frac{a_{1}+a_{2}}{a_{1}}\right)^{2 \nu-1} F\left(\nu+\frac{1}{2}, \nu+\frac{3}{2}, 2, \frac{a_{2}^{2}}{a_{1}^{2}}\right)
\end{aligned}
$$

(by Kummer's transformation).

This also shows that $g_{\nu}(\lambda)$ has no poles in the complex plane, and that (16) is an identity. By using Stirling's asymptotic expansion for the Gamma function in the series (13) which defines $b_{\nu}(\lambda)$, it may be shown that if $\nu$ is not a negative real,

limit $b_{\nu}(\lambda)=0$ (to the order $\nu^{-\frac{3}{2}}$ ) $|\nu| \rightarrow \infty$ 
Hence, if $\nu$ is not a positive real,

limit $b_{-\nu}(\lambda)=0$ (to the order $\left.\nu^{-\frac{3}{2}}\right)$

$|\nu| \rightarrow \infty$

Also since

limit $\cot \nu \pi=1$

$|\nu| \rightarrow \infty$

if $\nu$ is not real, it is evident from equation (15) that

limit $\cot \nu \pi b_{\nu}(\lambda)=0$ and limit $\cot \nu \pi b_{-\nu}(\lambda)=0$

$|\nu| \rightarrow \infty$

$|v| \rightarrow \infty$

if $\nu$ is not real.

In the equation (15) $\lambda^{-\nu}$ must be understood to represent the principal branch $e^{-\nu} \log \lambda$ where $\log \lambda$ is the real (natural) logarithm of $\lambda$. It is evident from (15) that if $\nu$ is not a pure real, $\operatorname{limit}_{|\nu| \rightarrow \infty} g_{\nu}(\lambda)=0$ if the real part of $\nu$ is negative, but $g_{\nu}(\lambda)$ becomes infinite with infinite $|\nu|$ if the real part of $\nu$ is positive. However, in this case $\underset{|\nu| \rightarrow \infty}{\operatorname{limit}} c^{\nu} g_{\nu}(\lambda)=0$ if the real part of $\nu$ is positive and $c$ is a positive real quantity less than $\lambda$.

If we desire a representation of the discontinuous function of $r$, namely, $\left[\frac{\partial M(r, \mu)}{\partial \mu}\right]_{\mu=0}$ as given by equation (12) in the form of an integral $\int f(\nu) r^{2 \nu} d \nu$ along some path in the complex $\nu$-plane, the equation (16) suggests that the poles of $f(\nu)$ must be the real integers, and it gives the value of the residues. No great intuition is then required to find that $\left(\frac{\partial M}{\partial \mu}\right)_{\mu=0}$ may be represented by the following: path integral in the $\nu$-plane

$$
\begin{gathered}
{\left[\frac{\partial M(r, \mu)}{\partial \mu}\right]_{\mu=+0}=\frac{a_{1}+a_{2}}{2 \pi i} \int_{i \infty}^{-i \infty}\left(\nu+\frac{1}{2}\right) g_{\nu}(\lambda)\left(\frac{r}{a_{1}+a_{2}}\right)^{2 \nu} d \nu} \\
=\frac{\pi\left(a_{1}+a_{2}\right)(1-\lambda)^{2}}{4 i} \int_{i \infty}^{-i \infty}\left(\nu+\frac{1}{2}\right) F\left(\frac{3}{2}, \nu+\frac{3}{2}, 3,1-\lambda\right)\left(\frac{r}{a_{1}+a_{2}}\right)^{2 \nu} d \nu \\
=\frac{\left(a_{1}+a_{2}\right)}{2 i} \int_{i \infty}^{-i \infty}\left(\nu+\frac{1}{2}\right) \cot \nu \pi\left\{b_{-\nu}(\lambda)\left(\frac{r}{a_{1}-a_{2}}\right)^{2 \nu}-b_{\nu}(\lambda)\left(\frac{r}{a_{1}+a_{2}}\right)^{2 \nu}\right\} d \nu
\end{gathered}
$$

where the path of the integral is down the entire imaginary axis of $\nu$ from $+i \infty$ to $-i \infty$ except that it crosses the real axis to the left of the origin and to the right of the point $\nu=-1$. In this integral $\left(\frac{r}{a_{1}+a_{2}}\right)^{2 \nu}$ $=e^{\nu \log \left(\frac{r}{a_{1}+a_{2}}\right)^{2}}$ where $\log \left(\frac{r}{a_{1}+a_{2}}\right)^{2}$ denotes the real (natural) logarithm.

To verify this induction consider first the case where $r>a_{1}+a_{2}$ so that $\operatorname{limit}_{\nu \mid \rightarrow \infty}\left(\frac{r}{a_{1}+a_{2}}\right)^{2 \nu}=0$ if the real part of $\nu$ is negative, in which case 
we have seen $g_{\nu}$ also vanishes. In this case we may make the path of the integral in equation (18) into a closed contour by adding to it a semicircular path of infinite radius, center at origin and lying to the left of the imaginary axis. There are no poles of the integrand in this contour, and on shrinking it to the vanishing point we conclude from equation (18) that

$$
\left(\frac{\partial M}{\partial \mu}\right)_{\mu=+0}=0 \text { if } r>a_{1}+a_{2}
$$

Consider next the case where $r<a_{1}-a_{2}$, and, hence, $0<\frac{r}{a_{1}+a_{2}}<\lambda$. In this case we have just seen that $\operatorname{limit}_{\nu \mid \rightarrow \infty}\left(\frac{r}{a_{1}+a_{2}}\right)^{2 \nu} g_{\nu}(\lambda)=0$ and also $\left(\nu+\frac{1}{2}\right)\left(\frac{r}{a_{1}+a_{2}}\right)^{2 \nu} g_{\nu}(\lambda)=0$ if the real part of $\nu$ is positive.

In this case we add a zero quantity to the integral in equation (18) by closing its path with an infinite semicircle lying to the right of the imaginary axis. As this contour contains no poles of the integrand, we deduce from equation (18) that

$$
\left(\frac{\partial M}{\partial \mu}\right)_{\mu=+0}=0 \text { if } r<a_{1}-a_{2}
$$

Finally, consider the case where $a_{1}-a_{2}<r<a_{1}+a_{2}$, so that $\frac{r}{a_{1}-a_{2}}>1$ and $\frac{r}{a_{1}+a_{2}}<1$.

Breaking up the integral as indicated in the last form of equation (18), the path of the integral

$$
\frac{a_{1}+a_{2}}{2 i} \int\left(\nu+\frac{1}{2}\right) \cot \nu \pi b_{-\nu}(\lambda)\left(\frac{r}{a_{1}-a_{2}}\right)^{2 \nu} d \nu
$$

may be similarly made into a closed contour encircling (in the negative direction) that part of the real axis to the left of the point $\nu=-1$ (inclusive), where all the poles of the integrand are the negative real integers. Since the integral $\mathcal{S} \cot \nu \pi d \nu$ taken in the negative direction around an infinitesimal loop encircling any one of these integers is just $-2 i$, this integral reduces to

$$
\begin{aligned}
& -\left(a_{1}+a_{2}\right) \sum_{k=-\infty}^{-1}\left(k+\frac{1}{2}\right) b_{-k}(\lambda)\left(\frac{r}{a_{1}-a_{2}}\right)^{2 k} \\
& =-\left(a_{1}+a_{2}\right) \sum_{k=-\infty}^{-1}\left(k+\frac{1}{2}\right) b_{k}(\lambda)\left(\frac{r}{a_{1}+a_{2}}\right)^{2 k}
\end{aligned}
$$


by equation (14). Similarly the path of the integral

$$
-\frac{a_{1}+a_{2}}{2 i} \int\left(\nu+\frac{1}{2}\right) \cot \nu \pi b_{\nu}(\lambda)\left(\frac{r}{a_{1}+a_{2}}\right)^{2 \nu} d \nu
$$

may be made a closed contour encircling (in the positive direction) all the positive real axis (including the origin). The residues from the poles of $\cot \nu \pi$, at $k=0,1,2,3 \ldots$ gives for this integral

$$
-\left(a_{1}+a_{2}\right) \sum_{k=0}^{\infty}\left(k+\frac{1}{2}\right) b_{k}(\lambda)\left(\frac{r}{a_{1}+a_{2}}\right)^{2 k}
$$

These two series give the development of $\left(\frac{\partial M}{\partial \mu}\right)_{\mu=0}=-2 \pi \rho \sigma$ by equation (12).

Consequently the problem to be solved is to find a function $M(r, \mu)$ which, in the range $0 \leqq \mu \leqq 1,0 \leqq r \leqq+\infty$, satisfies Laplace's equation, vanishes when $r \rightarrow \infty$ and also satisfies the boundary condition in the form given by equation (18). Since

$$
\nabla^{2}\left(r^{2 \nu} P_{2 \nu}(\mu)\right)=0 \text { where } P_{2 \nu}(\mu)=F\left(2 \nu+1,-2 \nu, 1, \frac{1-\mu}{2}\right)
$$

the solution of the problem is

or since

$$
M(r, \mu)=\frac{a_{1}+a_{2}}{2 \pi i} \int_{i \infty}^{-i \infty}\left(\nu+\frac{1}{2}\right) g_{\nu}(\lambda)\left(\frac{r}{a_{1}+a_{2}}\right)^{2 \nu} \frac{P_{2 \nu}(\mu)}{P^{\prime}{ }_{2 \nu}(0)} d \nu
$$

$$
P^{\prime}{ }_{2 \nu}(0)=-\frac{2 \sqrt{\pi}}{\Gamma\left(\nu+\frac{1}{2}\right) \Gamma(-\nu)}
$$

$$
\begin{aligned}
& M(r, \mu)=-\frac{a_{1}+a_{2}}{4 \pi^{3 / 2 i}} \int_{i \infty}^{-i \infty} \Gamma(-\nu) \Gamma\left(\nu+\frac{3}{2}\right) g_{\nu}(\lambda)\left(\frac{r}{a_{1}+a_{2}}\right)^{2 \nu} P_{2 \nu}(\mu) d \nu \\
& =\frac{a_{1}+a_{2}}{4 i \sqrt{\pi}} \int_{i \infty}^{-i \infty} \Gamma(-\nu) \Gamma\left(\nu+\frac{3}{2}\right) \cot \nu \pi P_{2 \nu}(\mu)\left\{b_{\nu}(\lambda)\left(\frac{r}{a_{1}+a_{2}}\right)^{2 \nu}\right. \\
& \left.-b_{-\nu}(\lambda)\left(\frac{r}{a_{1}-a_{2}}\right)^{2 \nu}\right\} d \nu
\end{aligned}
$$

If $0<\mu \leqq 1$, this integral defines a harmonic function, continuous with its derivatives, satisfying the boundary condition (18) at $\mu=+0$, and (as will be shown presently) one which vanishes when $r \rightarrow \infty$. A comparison of the last form of the integral (22) with the last form of (18) shows that we have introduced an additional factor

$$
\Gamma(-\nu) \Gamma\left(\nu+\frac{1}{2}\right) P_{2 \nu}(\mu)
$$


into the integrand, which introduces new poles, all on the real axis. Since $P_{2 \nu}(\mu)$ is defined as $F\left(2 \nu+1,-2 \nu, 1, \frac{1-\mu}{2}\right)$, it may be shown by Stirling's expansion that when $\nu$ is not real this new factor, when it becomes infinite with infinite value of $|\nu|$, does so in such a manner that its product by $\left(\frac{r}{a_{1}+a_{2}}\right)^{\nu}$ will vanish if the real part of $\nu$ is positive, for example, when $r<a_{1}+a_{2}$. Consequently it is possible in each case to make the same deformation of path for the integral (22) as was made in the corresponding case in discussion of the integral (18). We proceed to do this and to obtain the zonal harmonic expansions for $M$ in the three cases that arise.

\section{FORMULA WHEN THE DISTANCE BETWEEN CENTERS} IS GREATER THAN THE SUM OF THE RADII OF THE CIRCLES

$$
\text { If }\left(\frac{r}{a_{1}+a_{2}}\right)>1 \text {, then }\left(\frac{r}{a_{1}+a_{2}}\right)^{2 \nu} \rightarrow 0 \text { when }|\nu| \rightarrow \infty \text {, the real part of } \nu \text { being }
$$
negative. Hence, in this case we may swing the path of the integral in equation (22) to the left until it closes down upon the negative real axis to the left of and including the point $\nu=-1$. Here we meet the only poles of the integrand-those which make the factor $\Gamma\left(\nu+\frac{3}{2}\right)$ become infinite-namely, $\nu=-k-\frac{3}{2}$, where $k=0,1,2,3, \ldots$ Since $\frac{1}{2 \mu i} \int \Gamma\left(\nu+\frac{3}{2}\right) d \nu$ taken positively around an infinitesimal loop inclosing the point $\nu_{k}=-k-\frac{3}{2}$ is just $\frac{(-1)^{k}}{\Gamma(k+1)}$ if $k=0,1,2,3, \ldots$ the integral (22) reduces to

$$
M=\frac{a_{1}+a_{2}}{2 \sqrt{\pi}} \sum_{k=0}^{\infty}(-1)^{k} \frac{\Gamma\left(k+\frac{3}{2}\right)}{\Gamma(k+1)} g_{-k-\frac{3}{2}}(\lambda)\left(\frac{a_{1}+a_{2}}{r}\right)^{2 k+3} P_{-2 k-3}(\mu)
$$

But since $P_{\nu}(\mu)=P_{-\nu-1}(\mu)$ for all values of $\nu$

$$
P_{-2 k-3}(\mu)=P_{2 k+2}(\mu)
$$

Also by the identical property of $g_{\nu}(\lambda)$ expressed by equation (16)

$$
g_{-k-\frac{3}{2}}(\lambda)=\lambda^{k+\frac{3}{2}} g_{k+\frac{3}{2}}(\lambda)=\left(\frac{a_{1}-a_{2}}{a_{1}+a_{2}}\right)^{2 k+3} g_{k+\frac{3}{2}}(\lambda)
$$

Hence, for this case we find

$$
M(r, \mu)=-\frac{a_{1}+a_{2}}{2 \sqrt{\pi}} \sum_{k=1}^{\infty}(-1)^{k} \frac{\Gamma\left(k+\frac{1}{2}\right)}{\Gamma(k)} g_{k+\frac{1}{2}}(\lambda)\left(\frac{a_{1}-a_{2}}{r}\right)^{2 k+1} P_{2 k}(\mu)
$$


Inserting the two forms of $g_{k+\frac{1}{2}}(\lambda)$ given by equation (17) gives

$$
\begin{gathered}
M(r, \mu)=-\frac{\left(a_{1}+a_{2}\right) \pi^{3 / 2}(1-\lambda)^{2}}{4} \sum_{k=1}^{\infty}(-1)^{k} \frac{\Gamma\left(k+\frac{1}{2}\right)}{\Gamma(k)} \cdot \\
\cdot\left(\frac{a_{1}-a_{2}}{r}\right)^{2 k+1} P_{2 k}(\mu) F\left(\frac{3}{2}, k+2,3,1-\lambda\right) \\
=-\frac{\left(a_{1}+a_{2}\right) \pi^{3 / 2}(1-\lambda)^{2}}{4} \sum_{k=1}^{\infty}(-1)^{k} \frac{\Gamma\left(k+\frac{1}{2}\right)}{\Gamma(k)} \cdot \\
\cdot\left(\frac{a_{1}+a_{2}}{r}\right)^{2 k+1} P_{2 k}(\mu) F\left(\frac{3}{2}, 1-k, 3,1-\lambda\right)
\end{gathered}
$$

by the use of equation (16). Also

$$
\begin{gathered}
M(r, \mu)=-\frac{4 \pi^{3 / 2} a_{2}^{2}}{a_{1}} \sum_{k=1}^{\infty}(-1)^{k} \frac{\Gamma\left(k+\frac{1}{2}\right)}{\Gamma(k)}\left(\frac{a_{1}^{2}-a_{2}{ }^{2}}{a_{1} r}\right)^{2 k+1} \\
\cdot P_{2 k}(\mu) F\left(k+1, k+2,2, \frac{a_{2}{ }^{2}}{a_{1}^{2}}\right) \\
=-\frac{4 \pi^{3 / 2} a_{2}{ }^{2}}{a_{1}} \sum_{k=1}^{\infty}(-1)^{k} \frac{\Gamma\left(k+\frac{1}{2}\right)}{\Gamma(k)}\left(\frac{a_{1}}{r}\right)^{2 k+1} P_{2 k}(\mu) F\left(-k, 1-k, 2, \frac{a_{2}{ }^{2}}{a_{1}^{2}}\right)
\end{gathered}
$$

This is Butterworth's formula. The following form may also be of interest:

$$
\begin{aligned}
& M(r, \mu)=-\frac{4 \pi^{3 / 2} a_{1}^{2} a_{2}^{2}}{\left(a_{1}^{2}+a_{2}^{2}\right)^{\frac{3}{2}}} \sum_{k=1}^{\infty}(-1)^{k} \frac{\Gamma\left(k+\frac{1}{2}\right)}{\Gamma(k)}\left(\frac{a_{1}^{2}+a_{2}^{2}}{r^{2}}\right) \\
& \text { - } P_{2 k}(\mu) F\left(1-\frac{k}{2}, \frac{1}{2}-\frac{k}{2}, 2,\left(\frac{2 a_{1} a_{2}}{a_{1}^{2}+a_{2}^{2}}\right)^{2}\right)
\end{aligned}
$$

When the circles are nearly equal it is better to have coefficients which are expressible in ascending powers of $\lambda\left[=\left(\frac{a_{1}-a_{2}}{a_{1}+a_{2}}\right)^{2}\right]$. For this purpose we go back to (23a) and evaluate $g_{k+\frac{1}{2}}(\lambda)$ from its definition (15). It takes the indeterminate form $0 . \infty$ but is easily evaluated, and we find

$$
\begin{aligned}
g_{k+\frac{1}{2}}(\lambda) & =\operatorname{limit}_{\nu \rightarrow k+\frac{1}{2}\left[\lambda^{-\nu} b_{-\nu}(\lambda) \pi \cot \nu \pi\right]} \\
& =\frac{2 \pi^{\frac{3}{2}}(1-\lambda)^{2}}{\lambda^{k+\frac{1}{2}}} \frac{\Gamma\left(k+\frac{1}{2}\right)}{\Gamma(k+2)} F\left(\frac{3}{2}, 1-k, \frac{1}{2}-k, \lambda\right)
\end{aligned}
$$


so that (23a) takes the more convenjent form

$$
\begin{aligned}
& M(r, \mu)=-\pi\left(a_{1}+a_{2}\right)(1-\lambda)^{2} \sum_{k=1}^{\infty}(-1)^{k} \\
& \frac{\Gamma\left(k+\frac{1}{2}\right) \Gamma\left(k+\frac{1}{2}\right)}{\Gamma(k) \Gamma(k+2)}\left(\frac{a_{1}+a_{2}}{r}\right)^{2 k+1} P_{2 k}(\mu) F\left(\frac{3}{2}, 1-k, \frac{1}{2}-k, \lambda\right)
\end{aligned}
$$

Also Gauss' transformation changes (23d) to the form

$$
\begin{gathered}
M(r, \mu)=-\frac{2 \pi\left(a_{1}^{2}+a_{2}^{2}\right)}{r} \sum_{k=1}^{\infty}(-1)^{k} \frac{\Gamma\left(k+\frac{1}{2}\right) \Gamma\left(k+\frac{1}{2}\right)}{\Gamma(k) \Gamma(k+2)} . \\
\cdot\left(\frac{2\left(a_{1}^{2}+a_{2}^{2}\right)}{r^{2}}\right)^{k} P_{2 k}(\mu) F\left(-\frac{k}{2},-\frac{k+1}{2}, \frac{1}{2}-k,\left(\frac{a_{1}^{2}-a_{2}^{2}}{a_{1}^{2}+a_{2}^{2}}\right)^{2}\right)
\end{gathered}
$$

If the circles are equal, $a_{1}=a_{2}=a$ and $\lambda=\left(\frac{a_{1}-a_{2}}{a_{1}+a_{2}}\right)^{2}=0$, and these reduce to

$$
M(r, \mu)=-2 \pi a \sum_{k=1}^{\infty}(-1)^{k} \frac{\Gamma\left(k+\frac{1}{2}\right) \Gamma\left(k+\frac{1}{2}\right)}{\Gamma(k) \Gamma(k+2)}\left(\frac{2 a}{r}\right)^{2 k+1} P_{2 k}(\mu) \text { if } r>2 a
$$

\section{FORMULA WHEN THE DISTANCE BETWEEN CENTERS IS LESS THAN THE DIFFERENCE OF THE RADII}

In this case $\frac{r^{2}}{\left(a_{1}+a_{2}\right)^{2}}<\lambda$, hence $\operatorname{limit}_{|\nu| \rightarrow \infty}\left(\frac{r}{a_{1}+a_{2}}\right)^{2 \nu} g_{\nu}(\lambda)=0$ if the real part of $\nu$ is positive. This vanishes exponentially, so that if $\nu$ is not real, the additional factors $\Gamma(-\nu) \Gamma\left(\nu+\frac{3}{2}\right)$ do not prevent the integrand from vanishing. The path of the integral in (22) may, therefore, be shrunk to one encircling in a positive direction the positive real axis, including the origin. Here the poles of $\Gamma(-\nu)$ at $\nu=k=0,1,2,3 \ldots$ give the residues

$$
\frac{1}{2 \pi i} \int \Gamma(-\nu) d \nu=-\frac{(-1)^{k}}{\Gamma(k+1)}
$$

so that the integral (22) in this case reduces to

$$
M(r, \mu)=\frac{a_{1}+a_{2}}{2 \sqrt{\pi}} \sum_{k=0}^{\infty}(-1)^{k} \frac{\Gamma\left(k+\frac{3}{2}\right)}{\Gamma(k+1)} g_{k}(\lambda)\left(\frac{r}{\left(a_{1}+a_{2}\right)}\right)^{2 k} P_{2 k}(\mu)
$$


Or, using the two values of $g_{k}(\lambda)$ given in equation (17),

$$
\begin{gathered}
M(r, \mu)=\frac{\left(a_{1}+a_{2}\right) \pi^{\frac{3}{2}}(1-\lambda)^{2}}{4} \sum_{k=0}^{\infty}(-1)^{k} \frac{\Gamma\left(k+\frac{3}{2}\right)}{\Gamma(k+1)} . \\
\left(\frac{r}{\left(a_{1}-a_{2}\right)}\right)^{2 k} P_{2 k}(\mu) F\left(\frac{3}{2}, \frac{3}{2}-k, 3,1-\lambda\right)
\end{gathered}
$$

and

$$
\begin{aligned}
& M(r, \mu)=4 \pi^{\frac{3}{3}} \frac{a_{2}{ }^{2}}{a_{1}} \sum_{k=0}^{\infty}(-1)^{k} \frac{\Gamma\left(k+\frac{3}{2}\right)}{\Gamma(k+1} . \\
& \cdot\left(\frac{r}{a_{1}}\right)^{2 k} P_{2 k}(\mu) F\left(k+\frac{1}{2}, k+\frac{3}{2}, 2, \frac{a_{2}{ }^{2}}{a_{1}^{2}}\right)
\end{aligned}
$$

There is also the analogue of (23d),

$$
\begin{aligned}
& M(r, \mu)=\frac{4 \pi^{\frac{3}{2}} a_{1}^{2} a_{2}^{2}}{\left(a_{1}^{2}+a_{2}^{2}\right)^{\frac{3}{2}}} \sum_{k=0}^{\infty}(-1)^{k} \frac{\Gamma\left(k+\frac{3}{2}\right)}{\Gamma(k+1)}\left(\frac{r^{2}}{\left(a_{1}^{2}+a_{2}^{2}\right)}\right)^{k} . \\
& \cdot P_{2 k}(\mu) F\left(\frac{k}{2}+\frac{3}{4}, \frac{k}{2}+\frac{5}{4}, 2,\left(\frac{2 a_{1} a_{2}}{a_{1}^{2}+a_{2}^{2}}\right)^{2}\right)
\end{aligned}
$$

If $\lambda$ is small, the coefficients in (25a) may be computed by the following formula obtained by evaluating the $\operatorname{limit}_{\nu=k} g_{\nu}(\lambda)$ using (13), (14), and (15). This gives for $k=0,1,2,3 \ldots \ldots$

$$
\begin{aligned}
g_{k}(\lambda)= & -b_{k}(\lambda) \log \lambda+\sum_{s=-k}^{-1}(-1)^{s} \frac{\Gamma(-s) \Gamma\left(s-\frac{1}{2}\right) \Gamma\left(s+k-\frac{1}{2}\right)}{\Gamma(s+k+1)} \lambda^{s} \\
& -\sum_{s=0}^{\infty} \lambda^{s} \frac{\Gamma\left(s-\frac{1}{2}\right) \Gamma\left(s+k-\frac{1}{2}\right)}{\Gamma(s+1) \Gamma(s+k+1)}\left[\psi\left(s-\frac{1}{2}\right)+\psi\left(s+k-\frac{1}{2}\right)\right. \\
& -\psi(s+k+1)-\psi(s+1)]
\end{aligned}
$$

where $\psi(z)$ is defined in equation (29) below. The formula (25c) was derived by the writer in an earlier publication (see footnote 2 ). 


\section{FORMULA WHEN THE DISTANCE BETWEEN CENTERS} IS GREATER THAN THE DIFFERENCE AND LESS THAN THE SUM OF THE RADII

Consider first that part of the integral (22) which is

$$
-\frac{a_{1}+a_{2}}{4 i \sqrt{\pi}} \int_{i \infty}^{-i \infty} \Gamma(-\nu) \Gamma\left(\nu+\frac{3}{2}\right) \cot \nu \pi b_{-\nu}(\lambda)\left(\frac{r}{a_{1}-a_{2}}\right)^{2 \nu} P_{2 \nu}(\mu) d \nu
$$

Since $\frac{r}{a_{1}-a_{2}}$ is now $>1$, the path of this integral may be deformed into a closed contour encircling the negative real axis (in the negative or clockwise direction) and crossing the real axis between the origin and the point $\nu=-1$. The only poles of the integrand are the negative real integers, which are simple poles of $\cot \nu \pi$, for $b_{-\nu}$ has no poles on the negative real axis, and the poles of the factor $\Gamma\left(\nu+\frac{3}{2}\right)$ are the zeros of the factor cot $\nu \pi$.

Hence, since

$$
P_{-2 k}(\mu)=P_{2 k-1}(\mu)
$$

the above integral is given by

$$
\begin{aligned}
& -\frac{a_{1}+a_{2}}{4 i \sqrt{\pi}} \int_{i \infty}^{-i \infty} \Gamma(-\nu) \Gamma\left(\nu+\frac{3}{2}\right) \cot \nu \pi b_{-\nu}(\lambda)\left(\frac{r}{a_{1}-a_{2}}\right)^{2 \nu} P_{2 \nu}(\mu) d \nu= \\
& =-\frac{a_{1}+a_{2}}{2 \sqrt{\pi}} \pi \sum_{k=1}^{\infty}(-1)^{k} \frac{\Gamma(k)}{\Gamma\left(k-\frac{1}{2}\right)} b_{k}(\lambda)\left(\frac{a_{1}-a_{2}}{r}\right)^{2 k} P_{2 k-1}(\mu)
\end{aligned}
$$

.Next consider the remaining integral of (22), which is

$$
\frac{a_{1}+a_{2}}{4 i \sqrt{\pi}} \int_{i \infty}^{-i \infty} \Gamma(-\nu) \Gamma\left(\nu+\frac{3}{2}\right) \cot \nu \pi b_{\nu}(\lambda)\left(\frac{r}{a_{1}+a_{2}}\right)^{2 \nu} P_{2 \nu}(\mu) d \nu
$$

Since $\frac{r}{a_{1}+a_{2}}$ is here $<1$, the path of this integralmay be made to encircle (in a positive direction) the entire positive real axis (including the origin). On this side of the plane $b_{\nu}$ has no poles, nor has $\Gamma\left(\nu+\frac{3}{2}\right)$, but the positive integers (and zero) are now second-order poles of the factor $\Gamma(-\nu) \cot \nu \pi$. Since the integral

$$
\frac{1}{2 \pi i} \int \Gamma(-\nu) \cot \nu \pi f(\nu) d \nu
$$

taken around any one of these poles $\nu=k$, where $k=0,1,2,3 \cdots$, is just

$$
\frac{(-1)}{\pi}^{k+1}\left[\frac{d}{d \nu} \frac{f(\nu)}{\Gamma(\nu+1)}\right]_{\nu=k}
$$


it is found that

$$
\begin{aligned}
& \frac{a_{1}+a_{2}}{4 i \sqrt{\pi}} \int_{i \infty}^{-i \infty} \Gamma(-\nu) \Gamma\left(\nu+\frac{3}{2}\right) \cot \nu \pi b_{\nu}(\lambda)\left(\frac{r}{a_{1}+a_{2}}\right)^{2 \nu} P_{2 \nu}(\mu) d \nu= \\
& =-\frac{a_{1}+a_{2}}{2 \sqrt{\pi}} \sum_{k=0}^{\infty}(-1)^{k}\left[\frac{\partial}{\partial \nu}\left\{\frac{\Gamma\left(\nu+\frac{3}{2}\right)}{\Gamma(\nu+1)} b_{\nu}(\lambda)\left(\frac{r}{a_{1}+a_{2}}\right)^{2 \nu} P_{2_{\nu}}(\mu)\right\}\right]_{\nu=k}
\end{aligned}
$$

Adding equations (26) and (27), we find for the case where $a_{1}-a_{2}<$ $r<a_{1}+a_{2}$

$$
\begin{aligned}
\frac{2 \sqrt{\pi}}{a_{1}+a_{2}} M(r, \mu) & =-\pi \sum_{k=1}^{\infty}-(1)^{k} \frac{\Gamma(k)}{\Gamma\left(k-\frac{1}{2}\right)} b_{k}(\lambda)\left(\frac{a_{1}-a_{2}}{r}\right)^{2 k} P_{2 k-1}(\mu) \\
& -\sum_{k=0}^{\infty}(-1)^{k}\left(\frac{\partial}{\partial \nu}\left[\frac{\Gamma\left(\nu+\frac{3}{2}\right)}{\Gamma(\nu+1)} b_{\nu}(\lambda)\left(\frac{r}{a_{1}+a_{2}}\right)^{2 \nu} P_{2 \nu}(\mu)\right]\right)_{\nu=k}
\end{aligned}
$$

It is evident that each term of this series satifies Laplace's equation, since

$$
\nabla^{2} \frac{\partial}{\partial \nu} f(\nu) r^{2 \nu} P_{2 \nu}(\mu)=\frac{\partial}{\partial \nu} \nabla^{2} f(\nu) r^{2 \nu} P_{2 \nu}(\mu)=0
$$

In order to express this series in terms of functions which are suitable for computation, we may introduce the logarithmic derivative of the Gamma function which is usually denoted by $\psi(z)$ and which may be computed by the formula

$$
\psi(z) \equiv \frac{\Gamma^{\prime}(z)}{\Gamma(z)}=-c-\sum_{t=0}^{\infty}\left(\frac{1}{t+z}-\frac{1}{t+1}\right)
$$

where $c=0.57721566=$ Euler's constant. From the definition (13) of $b_{\nu}(\lambda)$, one finds

$\frac{\partial}{\partial \hbar} b_{k}(\lambda)=\sum_{s=0}^{\infty} \lambda^{s} \frac{\Gamma\left(s-\frac{1}{2}\right) \Gamma\left(s+k-\frac{1}{2}\right)}{\Gamma(s+1) \Gamma(s+k+1)}\left[\psi\left(s+k-\frac{1}{2}\right)-\psi(s+k+1)\right]$

Also

$$
\begin{aligned}
\frac{\partial}{\partial k} \frac{\Gamma\left(k+\frac{3}{2}\right)}{\Gamma(k+1)} & =\left[\psi\left(k+\frac{3}{2}\right)-\psi(k+1)\right] \frac{\Gamma\left(k+\frac{3}{2}\right)}{\Gamma(k+1)} \\
& =\frac{\Gamma\left(k+\frac{3}{2}\right)}{\Gamma(k+1)}\left[2 \log \frac{1}{2}+\frac{2}{2 \hbar+1}+\sum_{i=0}^{k-1 \geq 0}\left(\frac{1}{t+\frac{1}{2}}-\frac{1}{t+1}\right)\right]
\end{aligned}
$$


and

$$
\frac{\partial}{\partial k}\left(\frac{r}{a_{1}+a_{2}}\right)^{2 k}=\left(\frac{r}{a_{1}+a_{2}}\right)^{2 k} \log \left(\frac{r}{a_{1}+a_{2}}\right)^{2}
$$

These three formulas are valid for any value of $k$. It is well known that if $k$ is a positive integer or zero,

$$
\left[\frac{\partial P_{\nu}(\mu)}{\partial \nu}\right]_{\nu=k}=P_{k}(\mu) \log \left(\frac{\mu+1}{2}\right)+\psi_{k}(\mu)
$$

so that

$$
\left[\frac{\partial P_{2 \nu}(\mu)}{\partial \nu}\right]_{\nu=k}=2 P_{2 k}(\mu) \log \left(\frac{\mu+1}{2}\right)+2 \psi_{2 k}(\mu)
$$

where $\psi_{k}(\mu)$ is a polynomial in $\mu$ of degree $k$, which may be computed by either of the following formulas:

$\psi_{k}(\mu)=2(-1)^{k} \sum_{s=0}^{k}(-1)^{s}\left(\frac{\mu+1}{2}\right)^{s} \frac{(k+s) !}{s ! s !(k-s) !}[\psi(s+k+1)-\psi(s+1)]$

or

$\psi_{k}(\mu)=2(-1)^{k} \sum_{s=0}^{k-1}(-1)^{s} \frac{2 s+1}{(s+k+1)(s-7)}\left[P_{k}(\mu)-P_{s}(\mu)\right]$

The first form shows that $\psi_{0}(\mu)=0$ and the second that $\psi_{k}(1)=0$. (Since $\psi_{k}(\mu)$ is always written with a subscript, there is no danger of confusing these polynomials with the logarithmic derivative of the gamma function defined by equation (29). The notation in both cases seems well established by usage). Making use of formulas (30) to (33) in the series (28) gives

$$
\begin{aligned}
\frac{2 \sqrt{\pi}}{a_{1}+a_{2}} M(r, \mu) & =-\pi \sum_{k=1}^{\infty}(-1)^{k} \frac{\Gamma(k)}{\Gamma\left(k-\frac{1}{2}\right)} b_{k}(\lambda)\left(\frac{a_{1}-a_{2}}{r}\right)^{2 k} P_{2 k-1}(\mu) \\
& -\sum_{k=0}^{\infty}(-1)^{k} \frac{\Gamma\left(k+\frac{3}{2}\right)}{\Gamma(k+1)} b_{k}(\lambda)\left(\frac{r}{a_{1}+a_{2}}\right)^{2 k} P_{2 k}(\mu)\left[\frac{2}{2 k+1}\right. \\
& \left.+\sum_{t=0}^{k-1 \geq 0}\left(\frac{1}{t+\frac{1}{2}}-\frac{1}{t+1}\right)+2 \log \frac{r(\mu+1)}{4\left(a_{1}+a_{2}\right)}\right] \\
& -\sum_{k=0}^{\infty}(-1)^{k} \frac{\Gamma\left(k+\frac{3}{2}\right)}{\Gamma(k+1)}\left(\frac{r}{a_{1}+a_{2}}\right)^{2 k}\left[P_{2 k}(\mu) \frac{\partial b_{k}(\lambda)}{\partial k}\right. \\
& \left.+2 b_{k}(\lambda) \psi_{2 k}(\mu)\right]
\end{aligned}
$$


This formula gives the mutual inductance of the two parallel circles of radii $a_{1}$ and $a_{2}$ where $a_{2}<a_{1}$ for the case where $a_{1}-a_{2}<r<a_{1}+a_{2}$. Here $r$ is the distance between centers, $\mu=\cos \theta$ where $\theta$ is the angle between the common direction of their axes and the line connecting their centers, and $\mu$ is to be taken as positive. The quantity $\lambda$ is $\left(\frac{a_{1}-a_{2}}{a_{1}+a_{2}}\right)^{2}$, and the function $b_{k}(\lambda)$ is to be computed by formula and $\frac{\partial b_{k}(\lambda)}{\partial k}$ by formula (30); the $\psi$ function by (29) and the $\psi_{2 k}(\mu)$ by either of the formulas (34) where $k$ is to be replaced by $2 \%$.

It is not difficult to show that the three expansions here obtained for $M$ make $M$ and its derivatives continuous at the spherical surfaces $r=a_{1}-a_{2}$ and $r=a_{1}+a_{2}$ and make $\frac{\partial M}{\partial \mu}$ reduce to $-2 \pi r \sigma(r)$ when $\mu=+0$, and also make $M$ reduce in the first two cases, when $\mu=1$, to Maxwell's formula for coaxial circles. However, my direct proof that Maxwell's formula reduces to formula (35) when $\mu=1$ and $a_{1}-a_{2}<r<a_{1}+a_{2}$ is very complicated and is therefore left as an exercise for the reader. For the assistance of those interested, there follow a few relations valid for general value of $\nu$, which are derivable from the definition (13). They are

and

$$
\sum_{k=-\infty}^{\infty} \frac{b_{k}(\lambda)}{k-\nu}=-\pi \cot \nu \pi b_{\nu}(\lambda)
$$

Also

$$
\sum_{k=0}^{\infty} \frac{\Gamma\left(k+\frac{3}{2}\right)}{\Gamma(k+1)} \frac{\lambda^{k} b_{k}(\lambda)}{k+\nu}=-\pi \frac{\Gamma(\nu)}{\Gamma\left(\nu-\frac{1}{2}\right)} b_{\nu}(\lambda)
$$

$$
g_{\nu}(\lambda)=\frac{4 \pi\left(a_{1}+a_{2}\right)^{2 \nu-1}}{\nu+\frac{1}{2}} \int_{a_{1}-a_{2}}^{a_{1}+a_{2}} \rho^{-2 \nu} \sigma(\rho) d \rho \text { if } \nu \neq-\frac{1}{2}
$$

There is also a general relation for $0 \leqq \mu \leqq 1$ which may be useful

$$
\begin{gathered}
\sum_{n=0}^{\infty}(-1)^{n} \frac{\Gamma\left(n+\frac{1}{2}\right)}{\Gamma(n+1)} P_{2 n}(\mu) \frac{2 n+\frac{1}{2}}{\left(n+\frac{\nu}{2}+\frac{1}{2}\right)\left(n-\frac{\nu}{2}\right)}= \\
=-\frac{\pi}{\sin \frac{\nu \pi}{2}} \frac{\Gamma\left(\frac{\nu}{2}+\frac{1}{2}\right)}{\Gamma\left(\frac{\nu}{2}+1\right)} P_{\nu}(\mu)
\end{gathered}
$$


Returning to formula (35), consider the case where the circles are equal, $a_{1}=a_{2}=a$ and $\lambda=0$. Then

and

$$
b_{k}(0)=-2 \sqrt{\pi} \frac{\Gamma\left(k-\frac{1}{2}\right)}{\Gamma(k+1)}
$$

$$
\begin{aligned}
\frac{\partial b_{k}(0)}{\partial k} & =b_{k}(0)\left[\psi\left(k-\frac{1}{2}\right)-\psi(k+1)\right]= \\
& =-2 \sqrt{\pi} \frac{\Gamma\left(k-\frac{1}{2}\right)}{\Gamma(k+1)}\left[2 \log \frac{1}{2}-\frac{2}{2 k-1}+\sum_{t=0}^{k-1 \geq 0}\left(\frac{1}{t+\frac{1}{2}}-\frac{1}{t+1}\right)\right]^{(39)}
\end{aligned}
$$

This gives

$$
\begin{aligned}
M(r, \mu) & =4 a \sum_{k=0}^{\infty}(-1)^{k} \frac{\Gamma\left(k-\frac{1}{2}\right) \Gamma\left(k+\frac{3}{2}\right)}{\Gamma(k+1) \Gamma(k+1)}\left(\frac{r}{2 a}\right)^{2 k}\left\{\psi_{2 k}(\mu)+\right. \\
& \left.+P_{2 k}(\mu)\left[\log \frac{r(\mu+1)}{16 a}-\frac{2}{4 k^{2}-1}+\sum_{t=0}^{k-1 \geq 0}\left(\frac{1}{t+\frac{1}{2}}-\frac{1}{t+1}\right)\right]\right\}
\end{aligned}
$$

The first four terms of this series were given by Butterworth in the paper mentioned. (Footnote 1.)

The formula for unequal circles which are coplanar and which intersect is obtained by placing $\mu=0$ in equation (35). Since

it is found that

$$
P_{2 \nu}(0)=\frac{\cos \nu \pi}{\sqrt{\pi}} \frac{\Gamma\left(\nu+\frac{1}{2}\right)}{\Gamma(\nu+1)}
$$

$$
\begin{aligned}
2 \psi_{2 k}(0) & =P_{2 k}(0)\left[2 \log 2+\psi\left(k+\frac{1}{2}\right)-\psi(k+1)\right] \\
& =P_{2 k}(0) \sum_{t=0}^{k-1 \geq 0}\left(\frac{1}{t+\frac{1}{2}}-\frac{1}{t+1}\right)
\end{aligned}
$$

so that in this case

$$
\begin{aligned}
M(r, 0) & =-\frac{a_{1}+a_{2}}{2 \pi} \sum_{k=0}^{\infty}\left(\frac{r}{a_{1}+a_{2}}\right)^{2 k} \frac{\Gamma\left(k+\frac{1}{2}\right) \Gamma\left(k+\frac{3}{2}\right)}{\Gamma(k+1) \Gamma(k+1)}\left\{\frac{\partial b_{k}(\lambda)}{\partial k}+\right. \\
& \left.+2 b_{k}(\lambda)\left[\log \frac{r}{4\left(a_{1}+a_{2}\right)}+\frac{1}{2 k+1}+\sum_{t=0}^{k-1 \geq 0}\left(\frac{1}{t+\frac{1}{2}}-\frac{1}{t+1}\right)\right]\right\}
\end{aligned}
$$


Placing $a_{1}=a_{2}=a$ in this gives for equal coplanar, intersecting circles

$$
\begin{aligned}
M(r, 0) & =\frac{4 a}{\sqrt{\pi}} \sum_{k=0}^{\infty}\left(\frac{r}{2 a}\right)^{2 k} \frac{\Gamma\left(k-\frac{1}{2}\right) \Gamma\left(k+\frac{1}{2}\right) \Gamma\left(k+\frac{3}{2}\right)}{\Gamma(k+1) \Gamma(k+1) \Gamma(k+1)}\left\{\log \frac{r}{16 a}\right. \\
& \left.-\frac{2}{4 k^{2}-1}+\frac{3}{2} \sum_{t=0}^{k-1 \geq 0}\left(\frac{1}{t+\frac{1}{2}}-\frac{1}{t+1}\right)\right\}
\end{aligned}
$$

which is also the value given by equation (40) for $\mu=0$.

\section{SUMMARY}

The formulas $(23),(25)$, and (35), therefore, give the mutual inductance of any two parallel circles. It is believed that the formula (35) is the first formula yet given for this complicated case of unequal circles. The fact that $\frac{\partial M}{\partial x}$ is not continuous when $x \rightarrow 0$ and $a_{i}-a_{2}$ $<r<a_{1}+a_{2}$ shows the danger which lies in wait for one who constructs a solution of Laplace's equation and merely requires that it reduce to a known value upon a limited part of the axis of symmetry, without considering the boundary values at $x=0$.

Washington, February 20, 1929. 